The ASTROPHYSICAL Journal, 266:502-515, 1983 March 15

(1) 1983. The American Astronomical Society. All rights reserved. Printed in U.S.A.

\title{
THE DYNAMICS AND FUELING OF ACTIVE NUCLEI
}

\author{
COLIN NORMAN \\ Sterrewacht, Leiden, and Institute of Astronomy, Cambridge \\ AND \\ JOSEPH SILK \\ Department of Astronomy, University of California, Berkeley \\ Received 1981 November 16; accepted 1982 July 1
}

\begin{abstract}
Two outstanding problems are considered here that arise in quasar models in which the energy source is associated with the stellar fueling of a massive black hole in the nucleus of a galaxy. These problems are (1) the depletion of the loss cone, which, in a spherically symmetric potential, restricts the star capture rate to that allowed by two-body relaxation, and (2) the mechanism for stellar disruption: for a sufficiently massive black hole, stars are captured within a Schwarzschild radius without suffering tidal disruption and thereby yielding a usable fuel supply.

We show how a triaxial perturbation in the core of a galaxy containing a central massive black hole can very substantially increase the stellar fueling rate. The implication is that activity in galaxies may be strongly correlated with and, in fact, can be a direct consequence of such dynamical distortions in the central regions. We envisage such departures from symmetry to occur in the early stages of a galaxy's life, and perhaps later, as a consequence of merging, secular dynamical processes such as bar instabilities, or infall of massive gas clouds with a different angular momentum from the original core. Such dynamical processes can account for the entire range of observed activity in galactic nuclei and quasars.

If a massive accretion disk is present in the core, then the fueling rate can be additionally enhanced by the process of star-gas drag as the stars pass through the disk. The mechanism is especially important for massive black holes (above $\sim 10^{9} M_{\odot}$ ) where the tidal radius is inside the last stable orbit. The massive disk is heated by star-disk collisions, and for a Keplerian disk the characteristic spectrum over a finite radial extent is $S_{\nu} \propto \nu$. Dynamical studies of the symmetry of the inner regions of active galaxies preferably on scales below $\sim 100 \mathrm{pc}$ are most important for testing this type of model.
\end{abstract}

Subject headings: black holes - galaxies: nuclei - quasars

\section{INTRODUCTION}

Quasars and active galactic nuclei are generally believed to produce their prodigious luminosities via the release of gravitational energy associated with accretion and infall of matter onto a compact central object. For the purpose of the ensuing analysis, the central object will be assumed to be a massive black hole. While this assumption is not crucial to our discussion, the fact that a black hole provides the deepest possible central potential well does imply that it is the most natural candidate for the central engine. We will also assume that the quasar is associated with the nucleus of a conventional galaxy. The evidence for this is largely circumstantial but provides the basis for the only generally accepted class of quasar models.

The characteristic mass fueling rate for a quasar of luminosity $L$ is

$$
\dot{M} \approx L / \eta c^{2}=15(0.1 / \eta)\left(L / 10^{47} \mathrm{ergs} \mathrm{s}^{-1}\right) M_{\odot} \mathrm{yr}^{-1},
$$

where $\eta$ is the efficiency of conversion of infalling matter into observed radiation. If the lifetime $\tau$ of the quasar phase is $10^{8} \mathrm{yr}$, the mass of usable fuel consumed amounts to

$$
\sim 10^{9}(0.1 / \eta)\left(L / 10^{47} \mathrm{ergs} \mathrm{s}^{-1}\right)\left(\tau / 10^{8} \mathrm{yr}\right) M_{\odot} .
$$

This fuel requirement must be met in the innermost regions of the galaxy. 
It is by no means clear how a galaxy can provide this fuel reservoir. While luminous galaxies produce gaseous matter via stellar ejecta at a rate of $\sim 1 M_{\odot} \mathrm{yr}^{-1}$, very little (perhaps $1 \%-10 \%$ ) of this is available in the central bulge, let alone the galactic nucleus. Galactic winds are likely to be driven by supernovae and produce a net outflow of gas in the outer bulge region. The wind may be suppressed in the core (McDonald and Bailey 1981), resulting in a modest inflow that is capable of powering active nuclei, but inadequate for fueling quasars. Similar difficulties arise if gas injection from an external source, such as the infall of a gas-rich dwarf galaxy, is considered. In addition, the Eddington luminosity limit ultimately restricts spherically symmetric infall around a massive black hole: the limiting luminosity is $10^{47}\left(M_{h} / 10^{9} M_{\odot}\right)$ ergs s${ }^{-1}$, where $M_{h}$ is the mass of the central object. The principal problem that arises in constructing an inflow model is that it requires a sufficiently high column density in the infalling material so that it will not be affected by the radiation pressure-driven outflow of the forbidden-line-emitting gas. The evidence for outflow from Seyfert nuclei appears to be compelling (Heckman et al. 1981; Balick and Heckman 1982).

One can overcome most of these difficulties if stellar fueling provides the predominant energy source. Two new problems arise, however. First, the rate of stellar capture by the black hole must be sufficiently large. A potential difficulty arises in that once the loss cone is depleted, two-body relaxation may severely limit the stellar capture rate. Second, it is not sufficient to merely have the stars captured; they must be transformed into a usable form. Individual stars are swallowed without disruption by a sufficiently massive $\left(\geq 3 \times 10^{8} M_{\odot}\right)$ black hole (Hills 1975). Stellar destruction by collision is one possibility for overcoming this restriction; however, this requires an extreme stellar density within the central parsec that is well in excess of that obtained in evolutionary models of galactic nuclei (e.g., Sanders 1970). Stellar collisions and ensuing energy release dominate the dynamical evolution and rapidly deplete a dense core.

Our aim here is to discuss a simple scheme for resolving the difficulties that arise in a stellar fueling model of a massive black hole. Two aspects of the problem are developed below in some detail. In $\S$ II, we demonstrate how the loss cone may be refueled in a triaxial gravitational potential. We show that relatively small triaxial or nonaxisymmetric dynamical perturbations in the central region can be extremely significant. This is related to the lack of angular momentum conservation in such syștems for box orbits in particular, where, as will be shown in some detail later, for a departure from symmetry of order $\varepsilon$, these orbits will change their angular momentum on a time scale $(J / \dot{J}) \approx$ $\left(t_{\text {dyn }} / \varepsilon\right) \ll t_{R}$. Thus, the fueling rate can be significantly increased over the two-body estimate $t_{R}$ even for values of $\varepsilon \approx 1 \%-10 \%$. This effect was previously mentioned by Young (1977) for spherical cores distorted by rotational effects. Although the available fuel reservoir is no different from that in the symmetrical case, the triaxiality allows the possibility of a large boost in the fueling rate, thus providing a luminous but short-lived phase. Both the numerical models of Schwarzschild (1979) and Wilkinson and James (1982) for triaxial elliptical galaxies have values for $\varepsilon$ that very roughly amount to $\sim 1 \%-10 \%$ in the inner regions. This effect is not limited to spheroidal systems but can also apply to central barlike disturbances. Triaxiality is a common feature of the numerical collapse models of rapid rotators (van Albada 1982).

Simkin, Su, and Schwarz (1980) have argued that all Seyfert galaxies have bars or oval distortions in their central regions and present indirect evidence for this through an excess of observed ring structure morphology in Seyferts galaxies. Ring structures are clearly related to bar driving in the models of cloud orbits with collisions in a bar-driving potential (Schwarz 1981). Generally, Seyfert galaxies seem to be disturbed, have close companions, or are barred or ovally distorted. The evidence is not yet conclusive, but nonaxisymmetric effects could be playing a fundamental role here in forcing the fuel into the central engine. Additional evidence for such asymmetries in the central regions of active galaxies comes from beam precession inferred from radio source morphology. It is difficult to see how to precess a central massive black hole and its accretion disk by external tidal effects, and dynamical distortions in the central part seem necessary. Attractive possibilities are binary or even multiple massive black holes (Begelman, Blandford, and Rees 1980) which would not only precess the most massive disk but also coherently act to enhance the fueling rate due to violent scattering of stellar orbits.

Our second major theme is to study the stellar disruption induced by the interaction between stars and the dense gaseous environment around a massive black hole. Paczyński (1978) has argued strongly that such regions will be massive $\left(\sim 10^{9} M_{\odot}\right)$ accretion disks, self-gravitating in the direction normal to the disk and consequently of high column density at a radial distance $\leqslant 10^{16} \mathrm{~cm}$. Collapse to a disk can be inhibited, resulting in a quasi-spherical cloud heated by the star-gas drag of stars moving supersonically through the high column density accretion disk (BisnovatyKogan and Sunyaev 1972; Begelman and Rees 1978; Unno 1971; Hara 1978). Considering the detailed star-gas interaction in the gaseous disk, we demonstrate below that the star-gas drag heats the disk, which is then able to radiate away this energy. This results in a net loss of binding energy for the stellar system. Similar conclusions have been independently reached in a recent paper by $\mathrm{DaCosta}(1981)$. The stars can be trapped, refueling and heating the disk as they move radially inward, as far (in principle) as the last stable orbit around the black hole. This process of radiating the stellar binding energy could yield the quasar energy source. The assumption of self-gravitating disks and other 
possibly more dubious assumptions concerning the disk viscosity are not crucial to this model, which has the great advantage of being independent of disk viscosity but of giving similar effects.

The loss cone itself is now much larger than if it were determined by the tidal radius of the black hole, $r_{T} \approx 10^{14}\left(M_{H} / 10^{9} M_{\odot}\right)^{1 / 3} \mathrm{~cm}$. In fact, the loss cone includes stars within the radius at which they are trapped by the disk on a time scale

$$
\sim\left(\Sigma_{*} / \Sigma_{d}\right) t_{\mathrm{dyn}} \ll t_{R},
$$

where $\Sigma_{*}$ and $\Sigma_{d}$ are the column densities of a single star and the accretion disk, respectively, $t_{\mathrm{dyn}}$ is a characteristic dynamical time scale for the stellar orbits, and $t_{R}$ is the relaxation time scale. Straightforward implications are that the broad emission line filaments may be due to the star-disk interactions blowing off debris at a rate $\sim 1 M_{\odot} \mathrm{yr}^{-1}$, and that the continuum radiation is from a disk which is radiating the binding energy of a stellar cluster but which does not itself necessarily have to be viscous. The cutoff to the quasar luminosity noted by Hills (1975) when the tidal break-up radius moves inside the last stable circular orbit does not apply here. In fact, under the circumstances we envisage, it is an advantage that the stars can release all their binding energy due to star-gas drag until they are swallowed whole. Rough estimates of star-disk collisions are consistent with low-level quasar variability being triggered by such events. In more violent flares, the observed energy released in a flare is comparable to the entire quasar luminosity and must be stored in the disk, for example, by magnetic fields. However, the release could be triggered by star-gas collisions. Discussion of these points with a detailed analysis of stellar orbits and loss cone effects in triaxial systems comprises $\S$ II, followed by a study of gas inflow and disk formation in these triaxial or barred systems (§ III). Observational implications are discussed in $\S$ IV.

\section{FUELING IN A NONAXISYMMETRIC POTENTIAL}

As indicated in $\S \mathrm{I}$, the central regions may well be weakly triaxial, barred, or ovally distorted. We shall not here specifically consider the detailed origin of such nonaxisymmetric potentials, but there are at least three obvious possibilities. Galaxies may be formed as triaxial systems (see Binney 1979). This is consistent with the data on slowly rotating pressure-supported elliptical galaxies (see Illingworth 1981). Triaxiality is found in stable models of nonrotating and slowly rotating elliptical galaxies constructed by Schwarzschild $(1979,1981)$ and in numerical simulations for rapidly rotating triaxial systems formed in anisotropic collapse (Wilkinson and James 1982; van Albada 1982). In these cases, the orbits seem to contain a significant fraction $(\sim 5 \%)$ of low-order resonant orbits of, for example, the 1:1:1 type (i.e., orbits which can be described by harmonic oscillators about the center with approximately commensurable frequencies); this will become very relevant in our subsequent analysis.

Merging of galaxies will also result in an anisotropic velocity distribution due to preferential heating in the orbital plane of the merger. Simple estimates (Silk and Norman 1981) give a temperature anisotropy of $\sim 2: 1$ resulting from mergers of approximately equal mass galaxies. Transient bar configurations have been found in numerical simulations by Sanders and van Albada (1979) and Combes and Sanders (1981). These simulations lead to considerable velocity anisotropy as the bar dissolves, and the effects are quite sensitive to the stabilizing effects of the dark material. The possibility of such transient barlike perturbations as suggested by the above experiments is extremely intriguing, since it gives the possibility of turning the nonaxisymmetric driving force on and off. Bars may be generated either during formation or subsequent merging, and then may dissolve due to stochastic heating on a secular time scale $t_{s}$, where $t_{\mathrm{dyn}}<t_{s}<H_{0}^{-1}$.

Before proceeding to discuss the fueling rate analysis further, we turn to two related observable implications of a bar or triaxial potential, namely, the presence of ring structures in barred or ovally distorted systems and the common phenomenon of dust lanes and other infalling gaseous material orbiting around the major axis of a galaxy close to the plane containing the minor axis, perhaps the best known example being the Spindle galaxy.

The Spindle phenomenon (Shane 1979) appears to have a simple explanation in considering the stable periodic tubelike orbits in a triaxial potential (van Albada, Kotanyi, and Schwarzschild 1982; Binney 1981). The stable orbits are those about the largest and smallest axes, and those around the intermediate axis are unstable (Heiligman and Schwarzschild 1979). Depending on the initial trajectories of the infalling gas cloud, the gas will tend to settle down into one or other of the stable periodic orbits winding about the major or the minor axis or an annulus tilted out of the plane (Norman and Lake 1982). If the energy dissipation time scale is short compared to the angular momentum loss time scale, then the final state will be a disk about these periodic orbits. Gas may then be accreted in either of these two principal planes. One interesting possibility occurs if the gas fragments during infall and the fragments move on ballistic orbits suffering occasional collisions. The orbits will either be the above mentioned tube orbits circling the axis or box orbits which fill the region including the nucleus. Dissipative effects due to cloud-cloud collisions can allow box orbits to become highly centrally condensed, while the tube orbits could cool, dissipating their motion around the 
guiding center associated with the periodic orbits to form the narrow dust lanes commonly observed in elliptical galaxies (Norman and Lake 1982). Such dust lanes could be remnant indicators of infall, particularly those in the minor axis principal plane. Their effect may be to enhance fueling rates, and it is interesting that they are commonly found in active galaxies (Ekers 1981).

The generation of ring structures at the inner and outer Lindblad resonances of a disk galaxy can be formed by a bar or oval distortion acting on clouds moving in ballistic periodic orbits with dissipative collisions (Simkin, Su, and Schwarz 1980; Schwarz 1981). As shown in detail elsewhere, this can be understood in terms of test particle orbits with drag, where the drag is that due to big clouds colliding with small clouds in a Fokker-Planck approach (see Norman and Silk 1980; Cowie 1980). The clouds will move in epicyclic orbits lagging or leading the bar potential inside or outside corotation. If there is no inner Lindblad resonance, then for clouds inside corotation the dissipative collisions can allow clouds to give up their angular momentum to the bar and move toward the inner regions on a secular time scale of order 10 rotation periods. In strong bar simulations by Sellwood (1980), the pattern speed is sufficiently high that there is no inner Lindblad resonance, and strongly enhanced fueling can occur.

Of course, these statements can, in general, only be made with confidence for regions outside the very central regions within $100 \mathrm{pc}$ that we are considering here. Since we are assuming that the amount of triaxiality monotonically decreases to zero at the origin, the very modest $1 \%-10 \%$ triaxiality that we require at, say, $50-100 \mathrm{pc}$ will be a consequence of a larger value on kiloparsec scales.

Having somewhat labored the point, we now give the reason for its fundamental importance. In triaxial systems, angular momentum of each star is not conserved. As shown below, a typical box orbit, such as those found in the central parts of numerical models (de Zeeuw and Merritt 1982) will change its angular momentum on a time scale $t_{\mathrm{dyn}} / \varepsilon$, where $\varepsilon$ is a measure of the nonaxisymmetric perturbing component. Thus, the loss cone fueling problem can be substantially alleviated, since the time scale for repopulation of the loss cone is $t_{\mathrm{dyn}} / \varepsilon$, rather than that due to small angle scattering induced by relaxation effects $\sim\left(t_{\mathrm{dyn}} / t_{R}\right)^{1 / 2} t_{R}$; in other words, loss cone nonaxisymmetric feeding dominates if $\varepsilon \gg\left(t_{\mathrm{dyn}} / t_{R}\right)^{1 / 2}$.

We shall now sketch the results of a rigorous perturbation theory calculation about the origin of a nonrotating triaxial system. From a Taylor series expansion up to quartic terms, we have the Hamiltonian:

$$
H=\frac{1}{2}\left(x^{2}+y^{2}+z^{2}\right)+\frac{1}{2}\left(\omega_{1}^{2} x^{2}+\omega_{2}^{2} y^{2}+\omega_{2}^{2} z^{2}\right)+\varepsilon\left(\frac{1}{2} a_{1} x^{2} y^{2}+\frac{1}{2} a_{2} x^{2} z^{2}+\frac{1}{2} a_{3} y^{2} z^{2}+\frac{1}{4} b_{1} x^{4}+\frac{1}{4} b_{2} y^{4}+\frac{1}{4} b_{3} z^{4}\right),
$$

where the quartic terms are of order $\varepsilon$ compared to the quadratic terms. For box orbits in the central regions, the basic orbits are given in Cartesian coordinates by

$$
\begin{aligned}
& X=I_{1} \cos \theta_{1} \text { with } \theta_{1}=\omega_{1} t+\phi_{1} \text {, } \\
& Y=I_{2} \cos \theta_{2} \quad \theta_{2}=\omega_{2} t+\phi_{2}, \\
& Z=I_{3} \cos \theta_{3} \quad \theta_{3}=\omega_{3} t+\phi_{3} .
\end{aligned}
$$

Here $\omega_{1} \approx \omega_{2} \approx \omega_{3}$ in an almost spherical potential, and $\phi_{1}, \phi_{2}$, and $\phi_{3}$ depend on the initial conditions. The $I$ 's and $\theta$ 's are, in fact, the action angle variables. This is a general conditionally periodic motion for which each point in the box will undergo a sufficiently close passage of an individual stellar orbit after a significantly long, but finite, time (see Hagihara 1970). One finds that the rate of angular momentum change is given by

$$
\begin{aligned}
\frac{1}{J^{2}} \frac{d J^{2}}{\omega_{1} d t}= & {\left[\frac{\omega_{3}-\omega_{2}}{\omega_{1}} y_{0}^{2} z_{0}^{2} \sin 2\left(\theta_{3}-\theta_{2}\right)+\frac{\omega_{3}-\omega_{1}}{\omega_{1}} x_{0}^{2} z_{0}^{2} \sin 2\left(\theta_{3}-\theta_{1}\right)+\frac{\omega_{1}-\omega_{2}}{\omega_{1}} x_{0}^{2} y_{0}^{2} \sin 2\left(\theta_{1}-\theta_{2}\right)\right] } \\
& \times\left[y_{0}^{2} z_{0}^{2} \sin ^{2}\left(\theta_{3}-\theta_{2}\right)+x_{0}^{2} z_{0}^{2} \sin ^{2}\left(\theta_{3}-\theta_{1}\right)+x_{0}^{2} y_{0}^{2} \sin \left(\theta_{1}-\theta_{2}\right)\right]^{-1} .
\end{aligned}
$$

If, as is indeed the case here,

then

$$
\begin{gathered}
\frac{\omega_{1}-\omega_{2}}{\omega_{1}}, \quad \frac{\omega_{3}-\omega_{1}}{\omega_{1}}, \text { and } \frac{\omega_{1}-\omega_{2}}{\omega_{1}} \text { are of order } \varepsilon \\
\frac{1}{J^{2}} \frac{d J^{2}}{d t} \approx O\left(\omega_{1} \varepsilon\right)
\end{gathered}
$$


For tube orbits the situation is more complex and yet becomes simpler to discuss. There are two basic points to the reasoning. The first point is that for nearly spherical potentials in the central regions where $\omega_{1} \approx \omega_{2} \approx \omega_{3} \approx 1$, there are three integrals of motion: one exact, corresponding to the total energy of the system, and two asymptotic integrals, the first corresponding to the perturbed part of the Hamiltonian averaged over the unperturbed orbit, and the second which is evident in the numerical experiments and is probably related to a quantity which is a generalization of $J_{z}^{2}$. The latter two integrals are asymptotic in the sense that they are valid for time scales $\geq\left(\varepsilon^{2} \omega_{1}\right)^{-1}$, and in many cases $\gg\left(\varepsilon^{2} \omega_{1}\right)^{-1}$, as can be directly inferred from the numerical experiments. In other words, the tube orbits $\left(1 / J^{2}\right)\left(d J^{2} / d t\right)>\left(1 / \varepsilon^{2} \omega_{1}\right)$. Details will be given elsewhere (Norman 1982), but the problem we now face is that tube orbits are actual tubes fixed in space that do not pass through the center and do not change their orientation in time scales $<t_{\mathrm{dyn}} / \varepsilon^{2}$. The orbits will therefore not be involved in any population of loss cone orbits on significantly short time scales. The crucial question is then: what proportion of box orbits relative to tube orbits is present in the core regions? This proportion has to be high for the fueling process discussed here to work. In fact, this has been considered in detail in perturbation theory in the plane by de Zeeuw and Merrit (1982), who accurately estimated the radius (in Schwarzschild's numerical models) where the orbits changed from predominantly box to tube orbits. This is at several core radii! A similar analysis with similar results (de Zeeuw 1982) has been done for the three-dimensional rotating case. We can safely conclude that, inside the core region, the orbits are predominantly box orbits and the fueling mechanism still applies.

Assume now that the potential can be described by a spherically symmetric component and a small nonaxisymmetric component of order $\varepsilon$. The self-consistent calculation of how the asymmetry varies as one moves from the triaxial core to the spherically symmetric region near the black hole is extremely complicated. We shall adopt henceforth the simple parameterization $\varepsilon=\varepsilon_{0}\left(r / r_{h}\right)^{n}$, where $r_{h}=G m_{h} / \sigma^{2}$ and represents the radius of influence of the black hole in a core with velocity dispersion $\sigma$. We now use the result given above that the characteristic time scale for angular momentum to change in such a nonsymmetric potential is $\sim t_{\mathrm{dyn}} / \varepsilon$. Using this, we see that the effective angular scattering rate into the loss cone is $\sim t_{\mathrm{dyn}} / \varepsilon$, and this can be very much larger than the previously considered two-body scattering rate which occurs on a time scale $t_{R}\left(t_{\mathrm{dyn}} / t_{R}\right)^{1 / 2} \approx\left(t_{R} t_{\mathrm{dyn}}\right)^{1 / 2}$.

With $\varepsilon(r)=\varepsilon_{0}\left(r / r_{h}\right)^{n}, t_{\mathrm{dyn}} / \varepsilon$ is less than the characteristic time scale for small-angle scattering $\sim\left(t_{\mathrm{dyn}} t_{R}\right)^{1 / 2}$ in the region $r>r_{\varepsilon}$, where $r_{\varepsilon}$ is given by

$$
\frac{r_{\varepsilon}}{r_{h}} \approx\left[\frac{1}{\varepsilon_{0}^{2} N\left(r_{h}\right) \ln N\left(r_{h}\right)}\right]^{1 /(2 n-p+3)} \approx 10^{-28 / 13}\left\{\left[\frac{10^{10}}{N\left(r_{h}\right) \ln N\left(r_{h}\right)}\right]\left[\frac{10^{-3}}{\varepsilon_{0}^{2}}\right]\right\}^{4 / 13},
$$

where $N\left(r_{h}\right)$ is the total number of stars inside the sphere of influence of the black hole, and it is assumed that there is a cusp in the density profile $n(r)=n_{h}\left(r / r_{h}\right)^{-p}$. For $N\left(r_{h}\right)=10^{10}, p=7 / 4, \varepsilon_{0}^{2}=10^{-3}$, and $n=1$, we find $r_{\varepsilon} / r_{h}=10^{-3}$.

In the triaxial fueling region, the usual estimate of the critical radius of the loss cone is altered. Following the work of Frank and Rees (1976), we obtain the critical loss cone radius by setting the loss cone angle $\Theta_{\mathrm{lc}}=\left(f 2 r_{T} / 3 r\right)^{1 / 2}$ subtended at radius $r\left(r<r_{h}\right)$ by the tidal radius $r_{T}$ equal to the triaxial scattering angle in a dynamical time $\Theta_{D}=\varepsilon(r)$, where $f$ is a factor used to incorporate the effects of tidal capture into the cusp; for numerical estimates $f \approx 2$ unless otherwise specified. This yields

$$
\frac{r_{\text {crit }}}{r_{h}}=\left[\left(\frac{2 f}{3}\right)\left(\frac{r_{T}}{\varepsilon_{0}^{2} r_{h}}\right)\right]^{1 /(2 n+1)}=10^{-1}\left[\left(\frac{r_{T} / r_{h}}{10^{-5}}\right)\left(\frac{10^{-3}}{\varepsilon_{0}^{2}}\right)\right]^{1 / 3} \text { for } n=1 .
$$

Making the assumption that the velocity dispersion $\sigma$ is isotropic at $r_{\text {crit }}$, the fueling rate is

$$
S=\left[\pi n r^{2} \sigma\left(\pi \theta_{\mathrm{lc}}^{2}\right)\right]_{r=r_{\text {crit }}}=S_{0}\left[\left(\frac{2 f}{3}\right)\left(\frac{r_{T}}{\varepsilon_{0} r_{h}}\right)\right]^{-(2 p-1) / 2(2 n+1)},
$$

and putting in typical values, we find

$$
S / S_{0}=\left\{\begin{array}{lll}
10^{1.25}\left[\left(\frac{r_{T} / r_{h}}{10^{-6}}\right)\left(\frac{10^{-3}}{\varepsilon_{0}^{2}}\right)\right]^{-5 / 12} & \text { for } n=1, & p=7 / 4, \\
10^{0.84}\left[\left(\frac{r_{T} / r_{h}}{10^{-6}}\right)\left(\frac{10^{-3}}{\varepsilon_{0}^{2}}\right)\right]^{-1 / 4} & \text { for } n=2, & p=7 / 4,
\end{array}\right.
$$


where $S_{0}=2 \pi n_{c} v_{c} r_{h} r_{T}$ is the standard $n \sigma v$ value. The cusp law with pure triaxial fueling is still $p=7 / 4$ since only the angular momentum and not the energy is altered in this process, and the same scaling laws given by Shapiro and Lightman (1976) hold.

From these simple numerical estimates, we infer that even for small asymmetries in the core, the fueling rate can be very significantly boosted by factors of order $\sim 1-10$ which could change a normal nucleus swallowing $0.1-1 M_{\odot} \mathrm{yr}^{-1}$ into that of an active galaxy feeding off $1-10 M_{\odot} \mathrm{yr}^{-1}$.

\section{EFFECTS OF A MASSIVE ACCRETION DISK}

We have previously used a value of $r_{T}$ given by tidal effects. However, orbits can be trapped by the strong star-gas drag in dense self-gravitating Paczyński disks (Paczyński 1978) which have extremely high column densities, $\Sigma_{D}$. Typical values used here are $\Sigma_{D} \approx 10^{9} \mathrm{~g} \mathrm{~cm}^{-2}$ at $\sim 10^{16} \mathrm{~cm}$ from the central black hole. For the disk to trap an orbit that moves radially inward from within $r_{\text {crit }}$, it is necessary to dissipate orbital energy on a time scale $\sim t_{\text {dyn }} / \varepsilon$ before the orbit changes its angular momentum and moves out of the disk again. Assuming that a star's orbit will change if it sweeps up a fraction of order unity of its column density, we find that in order to be trapped, it must see a disk region with a column density $\Sigma_{\text {crit }}>\varepsilon \Sigma_{*}$, with the trapping time scale estimated to be

$$
t_{\text {trap }}=t_{\mathrm{dyn}}\left(\frac{\Sigma_{*}}{\Sigma_{D}}\right)<\frac{t_{\mathrm{dyn}}}{\varepsilon} .
$$

We shall now analyze the situation in more detail, examining in turn the new cusp law applicable to star-disk drag energy loss, the details of disk heating, the resulting radiation spectrum, the effect of stellar collisions, and finally its general importance for fueling a massive black hole.

\section{a) Stellar Distribution in the Cusp}

The mean orbital energy loss $\Delta\left(\frac{1}{2} m v^{2}\right)$ on one passage through the disk for an almost radial orbit making angle $\theta$ to the disk normal is given by

$$
\frac{\Delta\left(1 / 2 m v^{2}\right)}{1 / 2 m v^{2}}=\frac{\Sigma(R)}{\Sigma_{*}} \frac{1}{\cos \theta}
$$

where we henceforth use $r$ to denote distance of a star from the center and $R$ to denote distance in the disk from the center. Assuming that the box orbits fueling the loss cone have an isotropic velocity distribution, the number of stars per unit time at radius $r$ with mean energy $\frac{1}{2} m v^{2}(r)$ entering the loss cone subtended by a point on the disk at $r_{T}$ is

$$
S_{\mathrm{lc}} \approx \pi n(r) r^{2} v(r) f\left(\frac{2 R}{3 r}\right) \cos \theta \quad\left(r<r_{h}\right) .
$$

These stars will then lose a fraction $\Sigma(R) / \Sigma_{*} \cos \theta$ of their mean orbital energy $\frac{1}{2} m v^{2}(r)$. The energy lost due to star gas drag is then

$$
\left[\frac{1}{2} m v^{2}(r)\right] \pi n(r) r^{2} v(r) f(2 R / 3 r) \Sigma(R) / \Sigma_{*},
$$

independent of direction of impact. This flux is dissipated in the disk at radius $R$, giving a local heating to the disk which is assumed to be sufficiently dense to radiate all this heat input due to supersonic star-disk interactions. Since the velocity dispersion is assumed to be isotropic, we introduce an averaged value seen by a star with such a distribution:

$$
\langle R \Sigma(R)\rangle=\frac{1}{R_{\max }} \int_{R_{0}}^{R_{\max }} R \Sigma(R) d R,
$$

where $R_{0}$ and $R_{\max }$ are the inner and outer radii of the disk.

The energy flux is given by

$$
F_{*}=4 \pi r^{2} n(r)\left[\frac{1}{2} m v^{2}(r)\right] v(r) \frac{f}{6} \frac{\langle R \Sigma(R)\rangle}{r \Sigma_{*}}
$$


In a steady state set up presumably on time scale $\geq t_{R}$, we have a constant flux of stars and energy (Shapiro and Lightman 1976; Lightman and Shapiro; Bahcall and Wolf 1976), so that we can obtain the cusp law

$$
n(r)=\left(\frac{6}{f}\right)\left(\frac{F_{*}}{4 \pi r_{n}^{2} v\left(r_{h}\right)\left(\frac{1}{2} m v^{2}\left(r_{h}\right)\right)}\right)\left(\frac{\Sigma_{*} r_{h}}{\langle\Sigma(R) R\rangle}\right)\left(\frac{r}{r_{h}}\right)^{-3 / 2}
$$

which is consistent with the result for a central spherical gas cloud found independently by da Costa (1981).

\section{b) Disk Heating}

The mean heating rate of the disk by stellar drag at radius $R$ is then

$$
F_{\text {drag }}(R)=\frac{F_{0}}{2 \pi R^{2}}(R \Sigma(R) /\langle R \Sigma(R)\rangle) .
$$

This is primarily due to the slow energy dissipation of stars (Bisnovatyi-Kogan, Zel'dovich, and Novikov 1967) that are not completely trapped by the disk and are on very radial orbits when they pass through the disk. We shall consider below the case of fully trapped stars near the disk.

In the specific case of the Paczyński disk, the surface density is

$$
\Sigma(R)=\Sigma_{p_{0}}\left(\frac{R_{0}}{R}\right)^{3}\left[1-\left(\frac{R_{0}}{R}\right)^{1 / 2}\right] .
$$

Then the values $\langle R \Sigma(R)\rangle=1 / 3\left[\Sigma_{p_{0}} R_{0}^{2} / R_{\max }\right]$, and using $\Sigma_{p_{0}}=3 M_{d} /\left(2 \pi R_{0}^{2}\right)$, we find that

$$
F_{\text {drag }}(R)=\frac{F_{0}}{2 \pi R^{2}} \frac{1}{3}\left(\frac{R_{\max }}{R_{0}}\right)\left(\frac{R_{0}}{R}\right)^{2}\left[1-\left(\frac{R_{0}}{R}\right)^{1 / 2}\right] .
$$

Comparing the ratio of disk fueling rates due to star-disk drag (eq. [17]) averaged over the disk $\left\langle F_{\text {drag }}\right\rangle$ with that due to the tidal disruption mechanism (8), we find

$$
\begin{aligned}
\frac{\left\langle F_{\mathrm{drag}}\right\rangle}{\left\langle S m v^{2} / 2\right\rangle} & =10\left(\frac{M_{d}}{10^{9} M_{\odot}}\right)\left(\frac{10^{9} M_{\odot}}{M_{h}}\right)^{1 / 3}\left(\frac{10^{11} \mathrm{~g} \mathrm{~cm}^{-2}}{\Sigma_{*}}\right)\left(\frac{10^{16} \mathrm{~cm}}{R_{\max }}\right) \quad\left(r>r_{h}\right), \\
& =10\left(\frac{r}{r_{h}}\right)^{1 / 4}\left(\frac{M_{d}}{10^{9} M_{\odot}}\right)^{1 / 3}\left(\frac{10^{11} \mathrm{~g} \mathrm{~cm}^{-2}}{\Sigma_{*}}\right)\left(\frac{10^{16}}{R_{\max }}\right) .
\end{aligned}
$$

Disk-driven accretion of stars is therefore probably highly relevant when $M_{h} \gtrsim 4 \times 10^{8} M_{\odot}$, which is the mass range generally perceived to be necessary for producing quasar luminosities.

In making the above calculations using the Paczyński disk surface density, we have assumed that the heating effect of the stellar collisions does not alter the disk column density in the vertical direction. Consider now the parameter $A_{s}$ that Paczyński introduced to parameterize the ratio of the gravitational influence of the disk to that of the central black hole acting in the direction normal to the disk. The disk is self-gravitating when $A_{s}>1$. For a heating law $F \propto R \Sigma(R)$, $A_{s} \propto 1 / R$, and if $\Sigma(R) \approx R^{-3}, z_{0} \propto R$. Typical values quoted for $A_{s}$ in a normal Paczyński disk are $\sim 30$, and so our assumption that the disk structure is unchanged where the disk remains self-gravitating can be valid over a radial extent larger by a factor of $\sim 30$ than the initial radius at which stellar collisional heating becomes significant. More detailed estimates involving self-consistent structure of the disk are beyond the scope of this paper.

\section{c) Collisions}

The steady flow of stars and energy assumed hitherto can be depleted by collisions. The energy flow equation has a collisional sink term and is modified as follows:

$$
\frac{1}{r^{2}} \frac{\partial}{\partial r} F=-n^{2} \sigma_{*} v(r)\left[\frac{1}{2} m v^{2}(r)\right] .
$$


This can be solved to give

$$
n(r)=\frac{n\left(r_{h}\right)}{(1+C)\left(r / r_{h}\right)^{3 / 2}-C},
$$

where

$$
C=\left(\frac{3}{8 \pi^{3} f^{2}}\right) \frac{M\left(r_{h}\right)}{M_{d}}\left(\frac{R_{\max }}{r_{h}}\right) \ll 1
$$

There is a critical radius here where $n(r) \rightarrow \infty$ and the flow of stars cannot keep up with the collisional destruction. The physical meaning is that all stars will have been destroyed inside

$$
\left(\frac{r_{\mathrm{coll}}}{r_{h}}\right)=\left(\frac{C}{1+C}\right)^{2 / 3}
$$

assuming collisions are disruptive, as is expected for $r<r_{h}$, in general, for massive black holes $>10^{8} M_{\odot}$ (Frank and Rees 1976; Young, Shields, and Wheeler 1977; Frank 1978). A similar result can also be obtained by equating the inflow rate to the collisional rate. The fueling process envisaged here will occur when $r_{\text {coll }}$ falls inside $r_{\text {crit }}$. In the tidal disruption case, little is altered, but interesting effects may arise in the case of star-disk drag. Here the stars may be inhibited from orbiting into the black hole by collisions which will release material onto the disk. These trapped stars will be on circularised orbits because the maximum drag occurs almost impulsively at the distance of closest approach: this tends to reduce the eccentricity of an orbit. We consider therefore a simple model of this phase with circular orbits normal to the disk, since the angle-dependent terms cancel as shown previously. Calling $n(r, t)$ the stellar space density, we have

$$
\frac{\partial n}{\partial t}+v_{r} \frac{\partial n}{\partial r}=-n t_{\text {coll }}^{-1},
$$

where $v_{r}=d r / d t$ and $t_{\text {coll }}(r)$ is the stellar collision time scale. Assuming for calculational ease that all stars have circular orbits, with circular velocity $v(r)$, and that all orbits are normal to the disk, we see that the star-gas drag in the disk is described by

$$
\frac{d}{d t}\left(\frac{1}{2} M_{*} v^{2}\right)=\frac{-\Sigma(r) \sigma_{*} v^{3}}{\pi r},
$$

where $M_{*}$ and $\sigma_{*}$ are the mass and cross section of an individual star. It follows that

$$
v_{r}=\frac{2 \Sigma(r) \sigma_{*}}{\pi M_{*}}\left(\frac{G M_{h}}{r}\right)^{1 / 2} .
$$

If we assume that the collision time is given by

$$
t_{\text {coll }}^{-1}=n \sigma_{*} v_{\text {circ }},
$$

we find that

$$
\frac{\partial n}{\partial t}+\frac{2 \Sigma(r, t) \sigma_{*}}{\pi M_{*}}\left(\frac{G M_{h}}{r}\right)^{1 / 2} \frac{\partial n}{\partial r}=-n^{2} \sigma_{*}\left(\frac{G M_{h}}{r}\right)^{1 / 2} .
$$

The evolution of the disk surface density can be related to that of the stellar space density by assuming simply that stellar material released at $r$ moves within a radial shell and settles into the disk, adding to its surface density. Mass conservation then gives

$$
\frac{\partial \Sigma(r, t)}{\partial t} 2 \pi r d r=n^{2} \sigma_{*} v M_{*} 4 \pi r^{2} d r
$$


reducing to the evolution equation for $\Sigma$ :

$$
\frac{\partial \Sigma(r, t)}{\partial t}=2 r M_{*} \sigma_{*}\left(\frac{G M_{h}}{r}\right)^{1 / 2} n^{2}
$$

Rescaling the variables in these equations by putting $t \rightarrow t / t_{0}$, with $t_{0}=\left(G M_{h} / r_{0}^{3}\right)^{1 / 2}, \Sigma \rightarrow \Sigma /\left(M_{*} / \sigma\right), r \rightarrow r / r_{0}$, and $n \rightarrow n /\left(r_{0} \sigma_{*}\right)^{-1}$, we obtain

$$
\frac{\partial n}{\partial t}+2 \Sigma \frac{\partial n}{\partial r}=-r^{-1 / 2} n^{2}
$$

and,

$$
n=\frac{1}{2}\left(\frac{\partial \Sigma}{\partial t}\right)^{1 / 2}
$$

which yields the single nonlinear partial differential equation for $\Sigma$ :

$$
\Sigma_{\mathrm{tt}}+2 \Sigma \Sigma_{\mathrm{tr}}=r^{-1 / 2} \Sigma_{t}^{3 / 2}
$$

The solution to this nonlinear system is complex, and we shall at present restrict ourselves to solving the orbit trapping problem where the parameters of the disk are given. We take as our standard model the massive self-gravitating Paczyński disk, where the surface density $\Sigma_{p}$ is given by

$$
\Sigma_{p}=\left(\frac{r_{0}}{r}\right)^{3}\left[1-\left(\frac{r_{0}}{r}\right)^{1 / 2}\right]=\Sigma_{p_{0}}\left(\frac{r_{0}}{r}\right)^{3}\left[1-\left(\frac{r_{0}}{r}\right)^{1 / 2}\right] .
$$

The equation for the evolution of the stellar space density distribution is then

$$
\left\{\frac{\partial}{\partial t}+\Sigma_{p_{0}}\left(\frac{r_{0}}{r}\right)^{3}\left[1-\left(\frac{r_{0}}{r}\right)^{1 / 2}\right] \frac{\partial}{\partial r}\right\} n^{-1}=\left(\frac{r_{0}}{r}\right)^{\mathrm{i} / 2},
$$

which can be integrated along characteristics given by

$$
\Sigma_{p_{0}} \int\left(\frac{r_{0}}{r}\right)^{-3}\left[1-\left(\frac{r_{0}}{r}\right)^{1 / 2}\right]^{-1} d r=t+\text { constant } .
$$

The solution is

$$
n(r, t)=\frac{1}{\psi\left[t+2 r_{0} \Sigma_{p_{0}}^{-1} B_{\left(r / r_{0}\right)^{1 / 2}}(9,0)\right]-r_{0} \Sigma_{p_{0}}^{-1} B_{\left(r / r_{0}\right)^{1 / 2}}(9,0)},
$$

where the function $\psi$ is specified by initial conditions, namely, the distribution function at time $t=0$, as discussed in Appendix A, and where the incomplete gamma function $B_{\left(r / r_{0}\right)^{1 / 2}}(9,0)$ is defined in Abramowitz and Stegun (1964). A more specific calculation and example is given in Appendix $\mathrm{A}$, for $n(r, 0) \approx r^{-4}$ as an initial energy density profile. The principle result is that at large $r, n(r, t) \approx\left[t+c_{1}\left(r / r_{0}\right)^{4}\right]^{-1}$. At a fixed radius, stellar collisions deplete the core, with $n(r, t) \approx t^{-1}$.

\section{IMPLICATIONS}

\section{a) Time Variability}

We shall now study the variability in the disk luminosity caused by star-disk collisions assuming that the energy deposited in the disk is radiated. The details of the radiative transfer in the optically thick disk will not be discussed here. The assumption is that a burst will occur on a time scale short compared to the time between collisions at similar locations in the disk. 
The mean time between star-disk collisions within radius $R$ can be estimated in the $n \sigma v$ approximation to be

$$
\tau=\tau_{0}\left(R_{0} / R\right)
$$

where

$$
\tau_{0}=\left[2 \pi n_{c} v_{c} r_{h} R_{0}\right]^{-1}=0.613\left(\frac{10^{4} \mathrm{pc}^{-3}}{n_{c}}\right)\left(\frac{v_{c}}{300 \mathrm{~km} \mathrm{~s}^{-1}}\right)^{-1}\left(\frac{10^{9} M_{\odot}}{M_{h}}\right)^{-1} \mathrm{yr} .
$$

We have set

$$
R_{0}=\left(\frac{6 G M}{c^{2}}\right)=8.9 \times 10^{14}\left(\frac{M_{h}}{10^{9} M_{\odot}}\right) \mathrm{cm}
$$

and

$$
r_{h}=48\left(\frac{M_{h}}{10^{9} M_{\odot}}\right)\left(\frac{300 \mathrm{~km} \mathrm{~s}^{-1}}{v_{c}}\right) \mathrm{pc}
$$

There are two regions of interest. In the region where $\Sigma_{d}>\Sigma_{*}$, a star will be completely trapped and release its entire orbital energy. This will occur within a critical radius given by

$$
\frac{R_{\text {crit }}}{R_{0}}=\left(\frac{3 M_{d}}{2 \pi R_{0}^{2} \Sigma_{*}}\right)^{1 / 3}=2.3\left(\frac{M_{d}}{10^{9} M_{\odot}}\right)^{1 / 3}\left(\frac{10^{9} M_{\odot}}{M_{h}}\right)^{2 / 3}\left(\frac{10^{4} \mathrm{~g} \mathrm{~cm}^{-2}}{\Sigma_{*}}\right) .
$$

Within this radius the energy released in a burst is then

$$
E_{\text {burst }}=\frac{1}{6} M_{*} c^{2}\left(\frac{R_{0}}{R}\right)=3 \times 10^{53}\left(\frac{R_{0}}{R}\right) \mathrm{ergs},
$$

and we have a relation between the energy in a burst and the time between bursts given by

$$
E_{\text {burst }}=3 \times 10^{53}\left(\frac{\tau}{0.6 \mathrm{yr}}\right) \text { ergs. }
$$

At radii greater than $R_{\text {crit }}$, the energy released in a burst becomes

$$
E_{\text {burst }}=\frac{1}{6} M_{*} c^{2}\left(\frac{\Sigma_{p 0}}{\Sigma_{*}}\right)\left(\frac{R_{0}}{R}\right)^{4}=3.6 \times 10^{54}\left(\frac{R_{0}}{R}\right)^{4}\left(\frac{M_{d}}{10^{9} M_{\odot}}\right)\left(\frac{10^{9} M_{\odot}}{M_{h}}\right)\left(\frac{10^{11} \mathrm{~g} \mathrm{~cm}^{-2}}{\Sigma_{*}}\right) \mathrm{ergs},
$$

with the corresponding $E_{\text {burst }}-\tau$ relation:

$$
E_{\text {burst }}=1.3 \times 10^{53}\left(\frac{\tau}{100 \text { days }}\right)^{4} \text { ergs. }
$$

When sufficient variability data are available, it may be possible to test this type of model using an $E_{\text {burst }} \tau$ plot. By pushing the model to its extreme limits, one could in principle deduce the actual $\Sigma_{d}(R)$ from such a plot.

Although we do not consider in detail the star-gas interaction in the disk, we note that the minimum time scale of a burst $=10^{5}\left(R / R_{0}\right)^{3 / 2}\left(z_{0} / 0.1 R_{0}\right) \mathrm{s}$, where we use a scale height $z=0.1 R$. The postshock temperature at radius $r$ is very high $\left[\sim 2 \times 10^{10}\left(M_{h} / 10^{9} M_{\odot}\right)\left(10^{16} \mathrm{~cm} / R\right) K\right]$ and the star may be significantly ablated by heating of the stellar surface at a rate above the blackbody limit (e.g., Hara 1978). This will occur when the surface temperature of the star is

$$
T_{*}<10^{3}\left(\frac{M_{h}}{10^{9} M_{\odot}}\right)^{5 / 8}\left(\frac{10^{16} \mathrm{~cm}}{R}\right)^{11 / 8} \mathrm{~K} .
$$

At $R \leqq 10^{15} \mathrm{~cm}$, significant star-disk collisional mass injection may occur due to ablation from the surface. 
This model can be extended to explain the very largest amplitude variability seen in quasars. For such large variations, one requires an energy storage mechanism that can be released by stellar collisions with the disk. One possibility is to use the magnetic energy storage mechanisms discussed by Shields and Wheeler (1976), who show that at low accretion rates $\sim 0.1 M_{\odot} \mathrm{yr}^{-1}$, one can build up large energy reservoirs in magnetic fields of $>10^{52} \mathrm{ergs}$ at $\sim 0.1 r_{D}$. This will only be enhanced if a dynamo mechanism is acting in a turbulent accretion disk (Pudritz 1981). The relevant questions then are how could a stellar impact on the disk trigger such an outburst, and what are the consequences? In the related physics of solar flares, triggering mechanisms generally invoke the generation of anomalous resistivity in small localized volumes (Norman and Smith 1978), which then give rise to large-scale electric fields, particle runaway, and spreading of anomalous resistivity with associated sympathetic flaring.

As the star, assumed to be highly conducting, moves supersonically at $\sim 0.1 c$ within $\leq 10^{15} \mathrm{~cm}$ in a direction assumed (for simplicity) to be normal to the disk, very large electric fields will be generated, viz., $\left|\boldsymbol{v} \times \boldsymbol{B}_{D} / c\right| \approx 0.1 B_{D}$, where $B_{D}$ is the disk magnetic field. The bow shock of the star as it moves through the disk will give rise to microscopic instabilities. Both these actions will result in an anomalous resistivity that could act to trigger the magnetic energy in a flaring outburst.

\section{b) Emission Lines}

The basic constraints in the emission-line region are given by the absence of [O III], which yields a lower limit on the density, and the common occurrence of [C III], which imposes an upper bound on the density. High column densities are also derived by Canfield and Puetter (1980) who model the Ly $\alpha$ lines. Constrained by unobserved absorption effects, the filling factor $\Omega / 4 \pi$ is $\leq 10^{-2}$. This can be understood either in terms of filaments or of disk geometry. The line profiles in the broad-line region have been shown to be consistent with radiation-pressure-driven outflow (Blumenthal and Mathews 1975) or inflow (Cappriotti et al. 1980). For filamentary and turbulent models it is necessary to have a source of filaments. Proposed sources are radiative ablation of stars in the disk environments (Edwards 1980), stellar collisions, shock cooling and fragmentation, and, as we suggest here, disk disruption due to star-disk collisional interactions. No clear prediction emerges from the present models, although variability studies of specific species such as [O I] may eventually be able to provide a discriminant.

\section{c) Continuous Spectrum}

For a given surface brightness $F$, there is an effective temperature $T(R)=\left(F / 2 \sigma_{0}\right)^{1 / 4}$ at each point. Writing the energy dissipation rate in the disk in the general form

$$
F(R)=F_{0}\left(\frac{R_{0}}{R}\right)^{q}\left[1-\left(\frac{R_{0}}{R}\right)^{1 / 2}\right],
$$

and assuming that the disk radiates locally as a blackbody (Lynden-Bell and Pringle 1974), the spectrum is given by $S_{\nu} \propto \nu^{(3 q-8) / q}$. For an assumed Paczyński disk surface density, we have

$$
F(R)=\frac{1}{3} \frac{F_{0}}{2 \pi R^{2}}\left(\frac{R_{\max }}{R_{0}}\right) \frac{R \Sigma(R)}{\langle R \Sigma(R)\rangle}
$$

and therefore in this case $S_{\nu} \propto \nu$. For a viscous Keplerian disk, $p=3$, corresponding to $S_{\nu} \propto \nu^{1 / 3}$. For a Keplerian Paczyński disk heated by stellar impacts in radially plunging orbits, $p=4$ and $F_{\nu} \propto \nu^{1}$. For the Keplerian Paczyński disk heated by stars trapped by the disk, the power dissipation per unit area $\propto r^{-9 / 2} n(r)$, and if $n(r) \propto r^{1 / 2}, S_{\nu} \propto \nu$. If $S_{\nu} \approx \nu^{q}$ can be inferred from observation, then $n(r) \propto r^{-s}$, where $s=(9 q-11) /(3-q)$ for the case of trapped stars.

\section{d) Jet Precession Phenomenon}

In many instances (see Ekers et al. 1981), jets appear to swing through large angles with mirror symmetry on either side of the nucleus at a characteristic distance of $\sim 50-100 \mathrm{kpc}$ in the radio morphology. Clearly the precession must occur in the very central core region. The time scale roughly inferred from the radio data for the precession is typically $\sim 10^{8} \mathrm{yr}$. This is of the same order as the time scale we find for the angular momentum change of individual orbits in a triaxial potential. In models of the type discussed by Begelman, Blandford, and Rees (1980) and Roos (1981), consisting of a binary black hole nucleus formed as a result of merging, it is interesting to note that the stars can be scattered and heated by the hard binary via three-body encounters into an anisotropic velocity dispersion distribution in the core region. Furthermore, this hard binary can precess in a triaxial potential on $\sim t_{\mathrm{dyn}}\left(\sigma / v_{c}\right)^{3} \varepsilon^{-1}$, where $\left(\sigma / v_{c}\right)$ is a measure of the hardness of the binary. Another related possibility is that very massive disks with masses 
comparable to that of the black hole can themselves precess in a triaxial potential, eventually acting on the hole itself over long time scales via the Lense-Thirring effect (see Rees 1978; Begelman, Blandford, and Rees 1980). With a massive Paczyński disk, a very interesting mechanism for precessing the disk due to a triaxial potential can occur since the stars originating at a large distance of order $r_{h}$ from the disk and hitting the disk impart a triaxial torque to the disk due to the star-gas drag. Precessing jet sources should be closely examined for the effects of anisotropic velocity dispersion in the central regions, as well as for binary or multiple nuclei.

\section{e) Structure of Formerly Active Nuclei}

The ingredients of an active nucleus in the model given here are a black hole, a gas supply, and an asymmetric perturbation. When, then, is a quasar not a quasar? The black hole will not go away! Is the missing factor the gas supply or the lack of an asymmetric perturbing force? If such a perturbing force is absent, then enhanced fueling rates will turn off. Dead quasars should be of quite symmetric appearance. The massive Paczyński disks may be ultimately swallowed, or even turned into stars, if the disks, which are extremely optically thick, can undergo Jeans-type instabilities. Such very weak, small, cool, central stellar disks may signify the presence of a defunct quasar. If the disk remained gaseous and the mass fueling rates were very low, then if after some time, $M_{D} \gtrsim 1 / 3 M_{\mathrm{H}}$, the fueling process may regenerate itself again because such a self-gravitating disk is unstable to a bar-mode instability (Toomre 1981). The self-induced asymmetry could then act in the general way we have discussed to refuel the central engine. Inactive quasars, then, may be lying dormant awaiting the next triggering by internal bar mode or external merging to burst once more into active life!

In summary, our model predicts a definite correlation between stellar dynamical distortions and nuclear activity in galaxies. A triaxial perturbation in the central regions of a galaxy can substantially increase the fueling rate of a massive black hole in the nucleus. Typical fueling rates in a central cusp amounting to $0.1-1 M_{\odot} \mathrm{yr}^{-1}$ are boosted by a factor $\sim 10$ by triaxiality. A further consequence of triaxial fueling is that the inner regions of the cusp are continuously replenished even as stellar collisions deplete the core, resulting in a much more extended accretion for the luminous quasar phase than envisaged by McMillan, Lightman, and Cohn (1981). Nuclear activity in galaxies and in quasars can therefore be a direct consequence of dynamical distortions. The origin of such distortions may be identified with the initial collapse of the galaxy core or with subsequent secular dynamical bar instabilities, perhaps induced as a consequence of a merger. Finally, we emphasize that the key elements we have invoked to enhance the fueling rate of a central massive black hole, namely, dynamical distortions and the presence of a massive accretion disk, should be directly observable in the nuclei of nearby active galaxies.

We thank M. Begelman, G. Illingworth, G. Lake, C. McKee, M. Rees, N. Roos, and T. de Zeeuw for useful discussions. This work was completed at the 1981 CECAM galaxy workshop, and we thank C. Moser and J. Audouze for their hospitality and support. Travel between Berkeley and Leiden was partially supported by a NATO travel grant. The research of J. S. has been supported in part by the National Science Foundation under grant AST 79-15244 and by NASA under grant NGR05-003-578. J. S. also wishes to gratefully acknowledge the award of a Miller Professorship during the period that much of this research was performed.

\section{APPENDIX A}

\section{DETAILED EVOLUTION OF THE STELLAR DISTRIBUTION TRAPPED AROUND A PACZYŃSKI DISK}

The characteristics of equation (32) are given by

$$
\frac{d r}{d t}=\Sigma p_{0}\left(\frac{r_{0}}{r}\right)^{3}\left[1-\left(\frac{r_{0}}{r}\right)^{1 / 2}\right]
$$

which can be integrated to give

$$
t+2 r_{0} \Sigma_{p_{0}}^{-1} B_{\left(r / r_{0}\right)^{1 / 2}}(9,0)=\text { constant }
$$

The particular solution accounting for the source term on the right-hand side is obtained from

$$
\Sigma_{p_{0}}\left(\frac{r_{0}}{r}\right)^{3}\left[1-\left(\frac{r_{0}}{r}\right)^{1 / 2}\right] \frac{d f(r)}{d r}=\left(\frac{r_{0}}{r}\right)^{1 / 2}
$$


to be

$$
f(r)=r_{0} \Sigma_{p_{0}}^{-1} B_{\left(r / r_{0}\right)^{1 / 2}}(8,0),
$$

where the open beta-function $B_{x}(a, b)$ is defined in Abramowitz and Stegun (1964).

The solution of the evolution equation is found by writing it as

$$
\left\{\frac{\partial}{\partial t}+\Sigma_{p_{0}}\left[\left(\frac{r_{0}}{r}\right)^{3} 1-\left(\frac{r_{0}}{r}\right)^{1 / 2}\right] \frac{\partial}{\partial r}\right\}\left[n^{-1}(r)+f(r)\right]=0
$$

with

$$
f(r)=r_{0} \Sigma_{p_{0}}^{-1} B_{\left(r / r_{0}\right)^{1 / 2}}(8,0)
$$

In general,

$$
\left\{\frac{\partial}{\partial t}+\Sigma_{p_{0}}\left(\frac{r_{0}}{r}\right)^{3}\left[1-\left(\frac{r_{0}}{r}\right)^{1 / 2}\right] \frac{\partial}{\partial r}\right\} \psi=0
$$

if $\psi=\psi\left(t+2 r_{0} \Sigma_{p_{0}}^{-1} B_{\left(r / r_{0}\right)^{1 / 2}}(9,0)\right)$. Hence, it follows that

$$
n^{-1}+f(r)=\psi\left[t+2 r_{0} \Sigma_{p_{0}}^{-1} B_{\left(r / r_{0}\right)^{1 / 2}}(9,0)\right]
$$

giving

$$
n(r, t)=\frac{1}{\psi\left[t+2 r_{0} \Sigma_{p_{0}}^{-1} B_{\left(r / r_{0}\right)^{1 / 2}}(9,0)\right]-r_{0} \Sigma_{p_{0}}^{-1} B_{\left(r / r_{0}\right)^{1 / 2}}(8,0)},
$$

where $\psi$ is determined from

$$
\psi(q)=f\left[\hat{B}^{-1}(q)\right]-\frac{1}{n\left[\hat{B}^{-1}(q, 0)\right]}
$$

Here $\hat{B}=B_{\left(r / r_{0}\right)^{1 / 2}}(q, 0)$, and the inverse function can be obtained from tables. As an illustrative example, if $n(r, 0) \propto r^{-4}$, then $\psi(q) \propto q$, and

$$
n(r, t)=\frac{K}{t+2 r_{0} \Sigma_{p_{0}}^{-1}\left[B_{\left(r / r_{0}\right)^{1 / 2}}(9,0)^{-1 / 2} B_{\left(r / r_{0}\right)^{1 / 2}}(8,0)\right]},
$$

with $K$ constant. Clearly, more complicated evolutionary tracks may be found with different initial conditions.

\section{REFERENCES}

Abramowitz, M. M., and Stegun, I. A. 1964, Handbook of Mathematical Functions (New York: Dover), p. 263.

Bahcall, J. N., and Wolf, R. A. 1976, Ap. J., 209, 214.

Balick, B., and Heckman, T. M. 1982, Ann. Rev. Astr. Ap., in press.

Begelman, M., Blandford, R. D., and Rees, M. J. 1980, Nature, 287, 307.

Begelman, M. C., and Rees, M. J. 1978, M.N.R.A.S., 185, 847.

Binney, J. 1981, M.N.R.A.S., 196, 455.

Bisnovatyi-Kogan, G. S., and Sunyaev, R. A. 1972, Soviet Astr. - AJ, 16, 201.

Bisnovatyi-Kogan, G. S., Zel'dovich, Ya. B., and Novikov, I. D. 1967, Soviet Astr. - AJ, 11, 419.

Blumenthal, G. R., and Mathews, W. G. 1975, Ap. J., 198, 517.

Canfield, R. C., and Puetter, R. C. 1980, Ap. J. (Letters), 236, L7.

Cappriotti, E., Foltz, C., and Byard, P. 1980, Ap. J., 241, 903.

Combes, F., and Sanders, R. H. 1981, Astr. Ap., 96, 184.

Cowie, L. 1980, Ap. J., 96, 184.

Da Costa, L. N. 1981, M.N.R.A.S., 195, 869.

de Zeeuw, T., and Merritt, C. 1982, Ap. J., in press.

de Zeeuw, T. 1982, Proc. CECAM Workshop on Formation, Structure, Evolution of Galaxies, ed. J. Audouze and C. Norman, p. 11.

Edwards, A. C. 1980, M.N.R.A.S., 190, 757.

Ekers, R. D. 1981, in NATO Conf. on Normal Galaxies, ed. S. M. Fall and D. Lynden-Bell (Cambridge: Cambridge University Press), p. 169. 
Ekers, R. D., Fanti, R., Lari, C., and Parma, P. 1981, Astr. Ap., 101, 194

Frank, J. 1978, M.N.R.A.S., 184, 87.

Frank, J., and Rees, M. J. 1976, M.N.R.A.S., 176, 633

Hagihara, Y. 1970, Celestial Mechanics, Vol. 1 (Cambridge: MIT Press), pp. 132-137.

Hara, T. 1978, Progr. Theor. Phys., 60, 711.

Heckman, T. M., Wiley, G. K., van Breugel, W., and Butcher, H. R. 1981, Ap. J., 247, 403.

Heiligman, G., and Schwarzschild, M. 1979, Ap. J., 233, 872.

Hills, J. G. 1975, Nature, 254, 295.

Illingworth, G. 1981, in NATO Conf. on Normal Galaxies, ed S. M. Fall and D. Lynden-Bell (Cambridge: Cambridge University Press), p. 1.

Lightman, A. P., and Shapiro, S. L. 1977, Ap. J., 211, 244

Lynden-Bell, D., and Pringle, J. E. 1974, M.N.R.A.S., 168, 603.

McDonald, J., and Bailey, M. E. 1981, M.N.R.A.S., 197, 945.

McMillan, S. L., Lightman, A. P., and Cohn, H. 1981, Ap. J., 251, 436.

Norman, C. A., and Lake, G. 1982, Ap. J., submitted.

Norman, C. A., and Silk, J. 1980, IAU Symposium 87, Interstellar

Molecules, ed. B. H. Andrew, (Dordrecht: Reidel), p. 137.

Norman, C. A. 1982 , in preparation.

Norman, C. A., and Smith, R. A. 1978, Astr. Ap., 68, 145.

Paczyński, B. 1978, Acta. Astr., 28, 98.

Pudritz, R. 1981, M.N.R.A.S., 195, 881.
Rees, M. J. 1978, Nature, 275, 516.

Roos, N. 1981, Astr. Ap., 95, 349.

Sanders, R. H. 1970, Ap. J., 162, 791. Sanders, R. H., and van Albada, T. S. 1979, M.N.R.A.S., 189,
791.

Schwarz, P. 1981, Ap.J., 247, 77.

Schwarzschild, M. 1979, Ap. J., 232, 236. 1982 , in preparation

Sellwood, J. 1980, Astr. Ap., 89, 296

Shane, W. W. 1979, Astr. Ap., 82, 314

Shapiro, S. L., and Lightman, A. P. 1976, Nature, 262, 743.

Shields, G. A., and Wheeler, J. C. 1976, Ap. Letters, 17, 69

Silk, J., and Norman, C. A. 1981, Ap. J., 247, 59.

Simkin, S., Su, H., and Schwarz, P. 1980, Ap. J., 237, 404.

Toomre, A. 1981, in The Structure and Evolution of Normal Galaxies, ed. S. M. Fall and D. Lynden-Bell (Cambridge: Cambridge University Press), p. 111

Unno, W. 1971, Pub. Astr. Soc. Japan, 23, 123.

van Albada, T. S. $1982, M . N . R . A . S$. , in press.

van Albada, T. S., Kotanyi, C. G., and Schwarzschild, M. S. 1982 M.N.R.A.S., $198,303$.

Wilkinson, A., and James, R. 1982, M.N.R.A.S., 199, 171.

Young, P. J. 1977, Ap. J., 215, 36.

Young, P. J., and Shields, G. A., and Wheeler, J. C. 1977, Ap. J., 212, 367 .

Colin NoRman: Huygens Laboratory, University of Leiden, Wassenaarseweg 78, Leiden 2300 RA, Netherlands and Institute of Astronomy, Madingley Road, Cambridge CB3 OHA, England

JoSEPH SILK: Department of Astronomy, University of California, Berkeley, CA 94720 